\title{
Trombose venosa profunda: mitos e realidades
}

Susana Dias Antunes*

\section{RESUMO}

A trombose venosa profunda (TVP) é uma doença médica com uma incidência anual, nos EUA, de 117 casos por cada 100.000 pessoas, sendo uma das principais causas de morte nos doentes hospitalizados.

A sua etiologia é multifactorial, sendo necessária a interacção de vários factores para que exista a formação de um trombo no interior de um vaso sanguíneo.

O estabelecimento do grau de risco, o seu diagnóstico, e uma referenciação adequada aos cuidados de saúde secundários, para confirmação diagnóstica e tratamento, permite diminuir a mortalidade e morbilidade desta doença.

Neste artigo faz-se uma revisão sucinta sobre a etiologia, critérios de diagnóstico e tratamento da TVP.

Palavras-chave: Trombose Venosa Profunda; Anticoagulação; Heparinas de Baixo Peso Molecular, Síndroma Pós-Trombótico; Doença Venosa Crónica.

\section{INTRODUÇÃO}

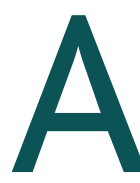
trombose venosa profunda (TVP) é um dos problemas médicos mais prevalentes, com uma incidência actual, nos EUA, de 117 casos em cada 100.000 pessoas. A nível global, calcula-se que apresente uma incidência estimada de cinco casos em cada 10000 pessoas. ${ }^{1}$ Este problema, bem como a sua sequela, o tromboembolismo pulmonar (TEP), são uma das principais causas de morte nos doentes hospitalizados nos Estados Unidos, estimando-se que cerca de 300.000 doentes morram, anualmente, por estas patologias. ${ }^{2}$

As primeiras definições de doença venosa datam de 1550 A.C e, em 1644, Schenk descreveu a oclusão da veia cava inferior, documentando, assim, o fenómeno de trombose profunda. Em 1864, Virchow reconheceu a associação entre TVP e TEP. ${ }^{2}$

A TVP resulta da oclusão do fluxo venoso dos membros por um coágulo sanguíneo, ao nível da circulação profunda. Na maioria dos casos, ocorre nos membros inferiores e, mais frequentemente, nas veias profundas da região do tornozelo e junto às válvulas, onde existe maior estase venosa por razões anatómicas, ou nas re-

*Assistente de Medicina Geral e Familiar, Unidade de Saúde Familiar Condes da Lousã, Agrupamento VII dos Centros de Saúde da Amadora. giões de confluência de vasos. Um número menor de casos ocorre no sistema ileofemoral por um mecanismo de lesão directa do vaso, como nos casos de cirurgias do foro ortopédico ou em procedimentos invasivos, como, por exemplo, a introdução de cateteres. ${ }^{2}$

O diagnóstico precoce, um reconhecimento das suas potenciais complicações e o tratamento adequado permitem evitar a morbilidade crónica e a morte a muitos doentes. $^{3}$

\section{BREVE REVISÃO ANATÓMICA DA CIRCULAÇÃO VENOSA DOS MEMBROS INFERIORES}

Dado que a maioria dos trombos se forma nos membros inferiores, efectua-se uma breve revisão anatómica da circulação venosa. Em termos práticos, a activação do sistema de coagulação ocorre em zonas de menor fluxo, nas cúspides valvulares ou nas confluências venosas.

O sistema venoso periférico funciona como um reservatório para o sangue excedente, bem como um condutor periférico, que conduz o sangue de volta para o coração e pulmões.

As veias mais periféricas são estruturas passivas, de parede fina, extremamente distensíveis. São, na sua maioria, supra-fasciais, pelo que podem acumular grandes quantidades de sangue sem interferência significativa na função venosa normal. Estas veias per- 
tencem ao sistema venoso superficial e o seu fluxo é recolhido através de veias mais profundas, com paredes mais espessas e menos distensíveis. As veias profundas são subfasciais, na sua maioria e rodeadas por tecidos densos de estrutura compacta.

O sistema venoso profundo dos membros inferiores engloba duas zonas: abaixo e acima do joelho. No tornozelo apresenta três grupos de veias, as tibiais anteriores (que drenam a região dorsal do pé); tibiais posteriores (drenam a região plantar) e peroneais (drenam a região lateral do pé).

A nível do joelho, todos estes sinusóides venosos, que formam o plexo solhar, se transformam na veia popliteia. Quando a veia popliteia passa através do canal dos aductores, na região posterior do joelho, passa a denominar-se veia femoral.

Na região proximal da coxa, a veia femoral une-se com a veia femoral profunda (que percorre a região lateral da coxa) para formar a veia femoral comum, que, ao passar na virilha, se torna na veia ilíaca externa. Esta, na região da articulação sacro-ilíaca, une-se à veia hipogástrica para formar a veia ilíaca comum.

A veia ilíaca comum esquerda é mais longa e oblíqua no seu trajecto, passando atrás da artéria ilíaca comum direita. Devido a esta assimetria anatómica pode existir compressão, por parte da artéria, sobre a veia ilíaca comum esquerda, com fibrose e proliferação da íntima, resultando no síndrome de May-Turner, associado a alguns casos de TVP.

Ao nível da 5. ${ }^{a}$ vértebra lombar, as duas veias ilíacas comuns unem-se para formar a veia cava inferior.

\section{ETIOLOGIA E FISIOPATOLOGIA DA TROMBOSE VENOSA PROFUNDA}

Virchow descreveu a presença de três factores que são de importância crítica para o aparecimento de trombose venosa: estase venosa, presença de lesão endotelial e activação do sistema de coagulação., ${ }^{2,3} \mathrm{~A}$ origem da TVP é, na maioria das vezes, multifactorial, o que foi demonstrado em múltiplos estudos epidemiológicos (Quadro I). ${ }^{1}$ A estase, por si só, não causa TVP, tal como estudos em animais evidenciaram, sendo necessário um estímulo adicional, como a lesão endotelial, para transformar um endotélio normalmente antitrombogénico num endotélio pró-trombótico, estimulando a produção de factores de coagulação. ${ }^{3}$

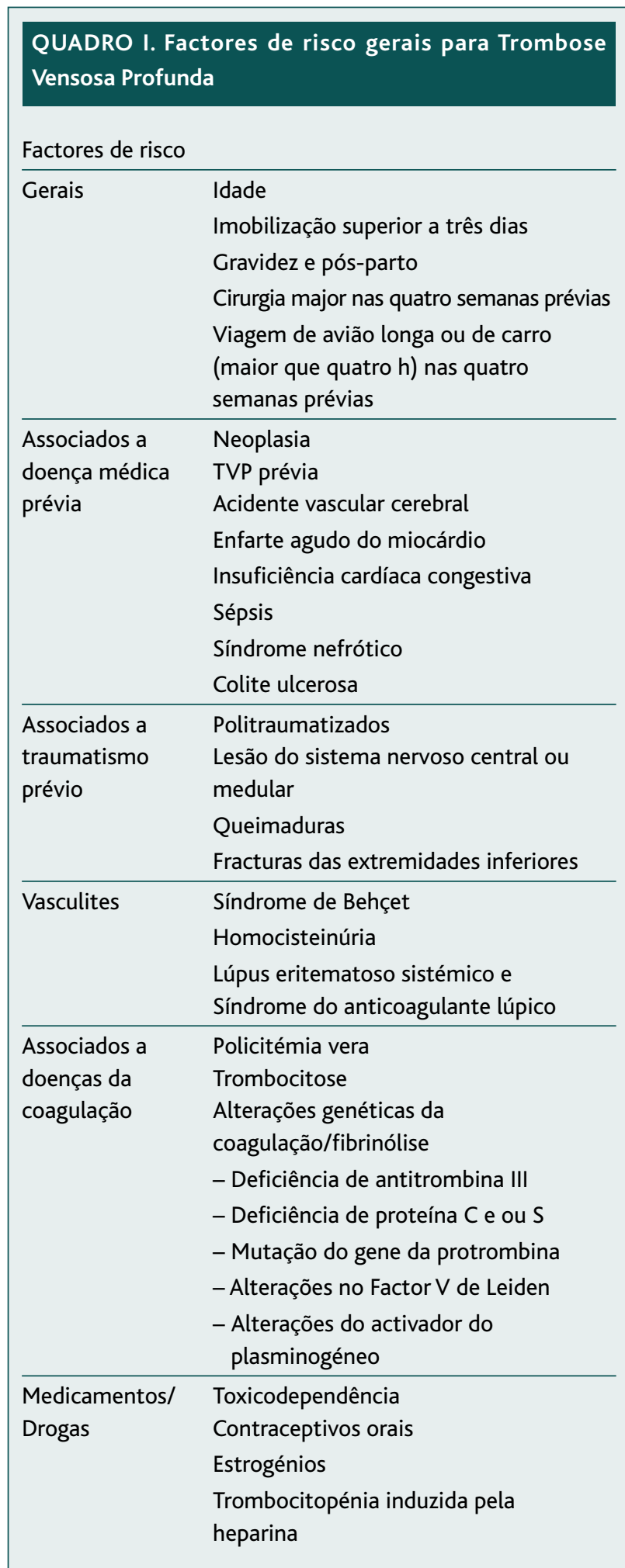


O factor de risco independente mais importante é a história prévia de TVP, que aumenta o risco de novo episódio em $25 \%$. Tal é demonstrado, na anatomo-patologia, pela presença frequente de restos de coágulos antigos nos trombos agudos. ${ }^{3}$

A idade é um dos factores de risco independentes, sendo que o risco aumenta cerca de $30 \%$ dos 30 aos 80 $\operatorname{anos}^{2,3}$

Existe uma relação bidireccional entre a doença neoplásica e a TVP, calculando-se uma incidência de 10-30\% de episódios de TVP nos doentes com neoplasia. As principais neoplasias associadas à TVP são as da mama, pulmão, próstata, cerebrais e síndromes mieloproliferativos. A fisiopatologia da TVP nestes doentes é também multifactorial, quer pela doença e pela imobilização prolongada, quer pelo uso de cateteres e de agentes citostáticos. Por outro lado, a TVP pode ser a primeira manifestação de uma neoplasia oculta. ${ }^{4}$

Nos doentes cirúrgicos é fácil perceber que o risco está aumentado, porque se trata de uma situação em que os três elementos da tríade de Virchow se encontram activos. A incidência depende, não só, do tipo de cirurgia (sendo maior nas cirurgias ortopédicas, oncológicas e do foro ginecológico) como do estado de saúde prévio do doente.

A gravidez e puerpério apresentam um risco associado de TVP de cerca de quatro a 14 vezes. Este aspecto deve--se às variações hormonais próprias da gravidez, que levam a um estado de hipercoagulabilidade, não só pelo aumento de fibrinogénio e de factorVIII, mas, também, pela diminuição dos níveis de fibrinólise e das proteínas C e S, mecanismo fisiológico que visa diminuir o risco de grandes hemorragias durante o parto. ${ }^{5}$

Em relação às viagens prolongadas, importa ressalvar que os dados publicados a este respeito são controversos. Os estudos que parecem demonstrar existir um aumento de risco de TVP, proporcionado pela imobilização e pela desidratação, geram dúvidas pela selecção de doentes neles utilizados. ${ }^{6}$

Uma ressalva particular deve ser feita para as mutações genéticas dentro do sistema da cascata de coagulação, que representam um dos factores de risco mais importantes para TVP. Estima-se que as deficiências primárias de antitrombina, proteína C e proteína S estejam associadas a cerca de $5-10 \%$ de todos os casos de TVP. A resistência dos factores pró - coagulantes a um sistema anticoagulante intacto foi recentemente descrita com a detecção da mutação do factor $V$ de Leiden, representando entre 10 a $65 \%$ de todos os doentes com TVP. $^{3}$

A trombofilia, entidade clínica definida como uma predisposição aumentada para a ocorrência de fenómenos trombóticos, tem como principal causa a origem genética, através da substituição de aminoácidos nas cadeias proteicas, criando proteínas estruturalmente anómalas. Factores não genéticos que podem causar trombofilia são, por exemplo, as alterações associadas à insuficiência renal, tratamento com metrotrexato e com antagonistas do ácido fólico. ${ }^{7}$

Factores que devem levantar a suspeita de se estar perante um caso de trombofilia, num doente com TVP, são: 7,8

- Idade inferior a 45 anos;

- História familiar de TVP;

- Trombose venosa em locais pouco habituais, como as artérias mesentérica, cerebral e hepática;

- TVP em doente correctamente anticoagulado;

- Púrpura fulminante do recém-nascido;

- História de abortos de repetição.

Nos doentes com suspeita de trombofilia deverá ser efectuado um estudo analítico dirigido (ver diagnóstico).

Um dos exemplos de trombofilia é o síndrome do anticorpo (Ac) antifosfolipídico, descrito em 1983 por Hughes, que se define como um estado trombifílico adquirido (arterial e/ou venoso) associado a abortos de repetição, presença de Ac anti-fosfolípido circulantes (anticoagulante lúpico ou anticardiolipina) e trombocitopénia. ${ }^{9}$

\section{HISTÓRIA CLÍNICA E EXAME FÍSICO}

Os sinais e sintomas de TVP relacionam-se com o grau de obstrução ao retorno venoso e de inflamação da parede do vaso. A grande maioria dos coágulos não provoca obstrução significativa ao fluxo e a inflamação do vaso pode ser mínima, pelo que o diagnóstico baseado apenas na história clínica é pouco preciso e pouco fiável. ${ }^{2,3}$

Alguns sinais e sintomas associados à doença são:

- Edema, principalmente unilateral, excepto se o trombo se localizar na bifurcação ilíaca, veias pélvicas ou veia cava, em que é bilateral; 
- Dor no membro atingido (cerca de 50\% dos doentes), por norma não relacionada com o tamanho, local ou extensão do coágulo;

- Desconforto e dor à palpação (cerca de 75\% dos doentes), principalmente na região média da perna, com dor à dorsiflexão do pé. O sinal de Hommans, pouco sensível e pouco específico, está presente em apenas $30 \%$ dos casos com TVP confirmada e pode aparecer em $50 \%$ dos casos sem TVP; 2

- Calor ou eritema cutâneo na área de trombose;

- Sinais de tromboflebite (cordão venoso endurecido, quente, palpável, resultado de lesão de veia do sistema superficial) estão presentes em cerca de $40 \%$ dos doentes com TVP;

- Phlegmasia cerúlea dolens: entidade rara, em que existe edema marcado, petéquias e cianose do membro, associada a obstrução maciça do sistema venoso ileofemoral;

- Phlegmasia alba dolens: entidade rara, designa uma situação em que existe obstrução maciça do sistema ileofemoral com vasoespasmo da artéria, resultando em palidez cutânea, petéquias, edema e ausência de pulsos distais.

Dado que o diagnóstico clínico de TVP é difícil, devido à relação entre os factores de risco e à natureza pouco específica dos sinais e sintomas, a utilização de uma escala de risco poderá ajudar o clínico na tomada de decisão. Uma das escalas de risco mais utilizadas é a deWells, que incorpora os factores de risco, sinais clínicos e presença ou ausência de diagnóstico alternativo (Quadro II). ${ }^{10}$

Os diagnósticos alternativos a considerar são:

- Celulite

- Tendinite Aquiliana

- Insuficiência arterial

- Artrite

- Edema periférico associado a insuficiência cardíaca congestiva; doença hepática, insuficiência renal ou síndrome nefrótico

- Hematoma

- Linfedema

- Lesão muscular ou dos tecidos moles

- Dor neuropática

- Rotura de quisto de Baker

- Veias varicosas

- Fracturas de stress ou outras lesões ósseas

\section{DIAGNÓSTICO LABORATORIAL E IMAGIOLÓGICO}

Os D-dímeros são produtos da degradação de fibrina que se encontram elevados em todas as situações médicas em que existe formação de coágulos, como, por exemplo, traumatismos, cirurgia recente, hemorragia, cancro e sepsis, bem como em situações de elevação da proteína $\mathrm{C}$, em doentes idosos.

O seu doseamento tem um papel importante no diagnóstico de TVP, mas, devido à sua baixa especificidade, este deve ser utilizado para excluir TVP e não para a sua confirmação. Os níveis permanecem elevados du-

\begin{tabular}{|c|c|}
\hline \multicolumn{2}{|c|}{$\begin{array}{l}\text { QUADRO II. Tabela de estratificação de risco de Wells para } \\
\text { a Trombose Venosa Profunda }\end{array}$} \\
\hline Parâmetros Clínicos & Pontuação \\
\hline $\begin{array}{l}\text { Cancro activo (com tratamento activo, ou } \\
\text { nos últimos seis meses, ou em cuidados } \\
\text { paliativos) }\end{array}$ & +1 \\
\hline $\begin{array}{l}\text { Paralisia ou imobilização recente das } \\
\text { extremidades inferiores }\end{array}$ & +1 \\
\hline $\begin{array}{l}\text { Imobilização no leito superior a três dias ou } \\
\text { cirurgia nas ultimas quatro semanas }\end{array}$ & +1 \\
\hline $\begin{array}{l}\text { Dor localizada ao longo da distribuição do } \\
\text { sistema venoso profundo }\end{array}$ & +1 \\
\hline Edema de todo o membro & +1 \\
\hline $\begin{array}{l}\text { Edema do tornozelo superior a três } \mathrm{cm} \text { em } \\
\text { comparação com o membro contralateral } \\
\text { assintomático }\end{array}$ & +1 \\
\hline $\begin{array}{l}\text { Edema com sinal de godet no membro } \\
\text { sintomático }\end{array}$ & +1 \\
\hline TVP prévia documentada & +1 \\
\hline $\begin{array}{l}\text { Presença de veias colaterais visíveis (não } \\
\text { varicosas) }\end{array}$ & +1 \\
\hline $\begin{array}{l}\text { Presença de diagnóstico alternativo } \\
\text { plausível }\end{array}$ & -2 \\
\hline \multicolumn{2}{|l|}{ Total } \\
\hline Elevada probabilidade & $>3$ \\
\hline Probabilidade moderada & 1 ou 2 \\
\hline Baixa probabilidade & 0 \\
\hline
\end{tabular}

Traduzido de Patel K, Feied C, Deep Venous Thrombosis, disponível em: http://emedicine.medscape.com, acedido em 13/07/2010, Actualizado em 16 de Junho de 2010. 
rante um período aproximado de sete dias. A evidência clínica actual sugere o uso do doseamento dos D-dímeros no algoritmo clínico de diagnóstico de TVP. Assim, em pacientes com doseamento negativo e com probabilidade baixa na escala de Wells, o diagnóstico fica excluído. Os doentes com doseamento positivo e risco moderado-alto na escala deWells devem efectuar um exame de imagem. ${ }^{2}$

O melhor sistema para efectuar o seu doseamento é por ELISA (Enzyme-Linked Immunoabsorbent Assay). ${ }^{1}$ Deve, no entanto, ter-se em conta que, embora a determinação dos D-dímeros permita, baseada numa estratificação de risco, estabelecer um diagnóstico de exclusão, nas pequenas TVP da região gemelar é onde este doseamento apresenta menos sensibilidade, especificidade e valor preditivo negativo. Os valores mais altos observam-se nas TVP dos sectores proximais. Valores considerados como positivos são superiores a 500 $\mathrm{ng} / \mathrm{ml}$ (maiores que 750 se em maiores de 70 anos), com método ELISA. ${ }^{1}$

No que respeita aos exames imagiológicos, o exame considerado gold-standard para o diagnóstico de TVP é a venografia de contraste. No entanto, por múltiplas razões, incluíndo reacções alérgicas, TVP induzida pelo próprio contraste, dificuldades técnicas, variabilidade entre observadores e custo, tem sido substituída por estudos não-invasivos, como a ultrassonografia venosa, que apresenta uma sensibilidade e especificidade, quando efectuada por observador experiente, de cerca de $95 \% .{ }^{2}$ No entanto, na região infragenicular, a mesma apresenta uma especificidade diminuída, de cerca de $50 \% .{ }^{11}$ A ultrassonografia permite, ainda, efectuar o diagnóstico diferencial com hematoma, ruptura de quisto de Baker, abcesso, ou outras causas de edema. A ressonância magnética nuclear é o teste imagiológico de escolha nos casos suspeitos de trombose da veia ilíaca ou da veia cava inferior. No segundo e terceiro trimestres de gravidez apresenta maior precisão diagnóstica que a ultrassonografia, devido à alteração provocada pelo útero no fluxo venoso. ${ }^{2}$

A atitude diagnóstica correcta inclui, não só, a confirmação da suspeita, mas, também, a exclusão de alguma causa, congénita e/ou adquirida que tenha podido desencadear a TVP, em particular em doentes jovens, com recorrência episódica ou com trombose em locais pouco comuns, que possam fazer suspeitar de trombofilia.
Outros estudos analíticos a considerar, especialmente na hipótese de trombofilia, devem incluir os doseamentos de proteína C, proteína S, do Factor V de Leiden, homocisteína, antitrombina III, FactorVIII, Factor IX, anticoagulante lúpico e Ac anti cardiolipina. Alguns autores defendem a execução deste estudo cerca de três - seis meses após o episódio de TVP, dada a possibilidade de alteração dos resultados pela reacção de fase aguda aquando do episódio., ${ }^{7,11,12}$ Também deve ser levado em conta que, nos doentes a efectuar terapêutica com dicumarínicos, estes devem ser interrompidos, dado que alteram os factores de coagulação dependentes da Vitamina K e as proteínas anticoagulantes referidas.

\section{ALGORITMO SIMPLIFICADO DE DIAGNÓSTICO}

Perante os factores clínicos e os exames já descritos, uma proposta de abordagem perante o doente será a descrita no algoritmo seguinte, adaptado com base na bibliografia referida: ${ }^{1,2}$

- Calcular a pontuação do doente na escala de Wells (Quadro II);

- Nos doentes com risco baixo: efectuar doseamento de D-dímeros. Se for negativo, exclui-se TVP, se positivo, deve efectuar-se ultrassonografia. Se o exame de imagem for sugestivo: tratar. Se for negativo, alguns autores consideram exclusão diagnóstica imediata, outros, reavaliação ao fim de uma semana com novo doseamento de D-dímeros e exame de imagem.

- Nos doentes de risco moderado, efectuar doseamento de D-dímeros. Se for negativo, exclui-se TVP. Se for positivo, efectuar exame de imagem e se este for positivo, tratar. Se o exame for negativo, o doente deve ser reavaliado clínica e imagiológicamente ao fim de uma semana.

- Nos doentes de elevado risco, efectuar directamente o exame de imagem. Se este for positivo, tratar. Se for negativo, efectuar doseamento de D-dímeros, e se o mesmo for negativo, excluir diagnóstico ou efectuar reavaliação clínica precoce (controverso). Se o doseamento de D-dímeros for positivo, efectuar venografia ou vigilância clínica e imagiológica, com repetição do exame imagiológico após uma semana. Caso se opte por efectuar venografia, se o resultado for sugestivo, tratar. 


\section{TRATAMENTO}

O tratamento tem como objectivo principal a prevenção do tromboembolismo pulmonar, diminuir a morbilidade e mortalidade e a prevenção do desenvolvimento da síndrome pós-trombótica.

A síndrome pós trombótica consiste no conjunto de sinais e sintomas que resultam da obstrução crónica do fluxo venoso e da lesão valvular, juntamente com alterações da microcirculação a nível local. A sua incidência após um episódio confirmado de TVP varia entre 20 e $50 \%$, dependendo dos estudos, sendo que entre estes existe pouca uniformidade nos desenhos dos mesmos, follow-up sintomatológico e tempo de seguimento. Os sintomas associados incluem dor crónica, eritema, edema e úlceras cutâneas. ${ }^{13,14}$

\section{a) Tratamento inicial da TVP:}

O tratamento base da TVP consiste na anticoagulação. Esta pode ser efectuada, inicialmente, através da administração de heparina não fraccionada por via endovenosa (tratamento clássico) ou através das novas heparinas de baixo peso molecular, e continuada, posteriormente, com anticoagulantes orais, ou mantendo as heparinas de baixo peso molecular, de forma a prevenir as recorrências.

Por norma, o tratamento anticoagulante inicial deve ser feito com heparina não fraccionada ou heparina de baixo peso molecular, durante pelo menos cinco dias, e depois continuado com dicumarínicos. Nos primeiros dias de tratamento com dicumarínicos, deve ser mantida a heparina, até o INR ser superior a 2 , para minimizar o risco de hipercoagulabilidade inicial induzido pela varfarina. Posteriormente, após pelo menos $48 \mathrm{~h}$ de INR alvo mantido, pode então suspender-se a heparina e manter apenas o fármaco dicumarínico. ${ }^{14,15}$

\section{1) Heparina não fraccionada}

A heparina não fraccionada apresenta um peso molecular entre 3000 e 30000 Da e a sua actividade antocoagulante depende da união de um pentassacárido à antitrombina III, potenciando a inibição da trombina e do factor X activado (Xa). Apresenta, ainda, uma forte afinidade por diversas proteínas plasmáticas, como o factor de Von Willebrand, as plaquetas, os macrófagos e as células endoteliais, o que limita a sua capacidade anticoagulante. ${ }^{14}$
A sua utilização é menos prática que a das heparinas de baixo peso molecular, que apresentam doses ajustadas ao peso e não necessitam de controlo laboratorial. A sua administração implica o internamento obrigatório do doente e a realização de controlos laboratoriais para monitorização da dose, dado que a resposta é bastante variável de doente para doente. A sua monitorização é feita pelo doseamento de APTT (tempo de tromboplastina parcial activado), que deve manter-se pelo menos uma vez e meia superior ao normal. É o tratamento de eleição nos doentes com IRC. ${ }^{2,3}$

As principais complicações associadas a este tratamento são as hemorragias, a trombocitopénia e a osteoporose. O fenómeno de trombocitopénia ocorre em cerca de 1 a $2 \%$ de todos os doentes, entre o quinto e o décimo dia de tratamento, e em cerca de 0,1 a $0,2 \%$ dos casos ocorre por fenómenos de auto-imunidade mediada por anticorpos IgG, que podem levar a trombose arterial ou venosa, com complicações tais como a amputação ou morte. A ocorrência de trombocitopénia mediada pela heparina implica a suspensão imediata da mesma, continuando-se a anticoagulação com agentes heparinóides específicos (p.e. argatroban)..$^{14,16}$

\section{2) Heparinas de baixo peso molecular}

A fragmentação da heparina por métodos enzimáticos permitiu o desenvolvimento de novas moléculas, as heparinas de baixo peso molecular. Estas são administradas por via sub-cutânea, o que permite o máximo efeito anti-Xa ao fim de três - quatro horas. O seu efeito anticoagulante baseia-se na união à antitrombina III bem como numa grande capacidade de inactivação do factor Xa.

Ao ter menor peso molecular, apresenta, também, menor afinidade pelas diferentes proteínas plasmáticas, o que se repercute nas suas propriedades terapêuticas, permitindo uma resposta anticoagulante previsível a doses fixas e ajustada ao peso corporal, maior biodisponibilidade e eliminação puramente renal, bem como semi-vida mais longa. A monitorização da dosagem não é consensual, sendo considerada desnecessária na maioria dos doentes, excepto nas grávidas e na insuficiência renal crónica grave. ${ }^{15}$ Esta pode ser feita pelo intervalo terapêutico de factor anti Xa, que, pode, no entanto, não estar disponível em todos os hospitais. ${ }^{14} \mathrm{O}$ doseamento deve ser efectuado, idealmente, 
quatro horas após a administração e os valores terapêuticos devem encontrar-se entre 0,6 e $1 \mathrm{UI} / \mathrm{ml}$, se a administração é feita cada 12 horas como é recomendado. ${ }^{14,15}$

Vários ensaios clínicos compararam os regimes de administração diária com os de administração bi-diária para detectar diferenças em termos de segurança e eficácia. Na sua maioria, verificou-se que a administração única diária era sobreponível à bi-diária, mas persiste alguma preocupação se a administração única não acarreta maior risco de recidivas, pelo que, na sua maioria, se utiliza a administração em duas tomas diárias, sobretudo nos doentes com maior risco de recidiva ou hemorragia: idosos, grávidas, doentes com cancro e doentes com TVP recorrente..$^{14,15}$

Nas grávidas devem ser utilizadas apenas as heparinas de baixo peso molecular. No caso particular dos doentes com obesidade mórbida, e apesar dos estudos não terem demonstrado uma incidência relevante de episódios hemorrágicos quando administrada a dose em função do peso real, deve ter-se em conta o doseamento dos níveis de Ac anti factor Xa cerca de quatro horas após a administração subcutânea, para verificação do intervalo terapêutico. ${ }^{2}$

Algumas semelhanças e diferenças entre as heparinas não fraccionadas e as de baixo peso molecular encontram-se descritas no Quadro III. No Quadro IV encontram-se descritas as doses habituais recomendadas para as heparinas de baixo peso molecular, no tratamento inicial da TVP.

\section{3) Outras terapêuticas existentes}

- Terapêutica trombolítica: associada a menor risco de recorrência e a menor risco de síndrome pós-flebítico. Apresenta indicações restritas, nomeadamente em relação com o tipo e extensão de trombo, para além do risco relativo de hemorragia estar bastante aumentado neste tipo de procedimento. Actualmente está reservada praticamente para situações agudas de trombose ileo-femoral. Não existe evidência clínica actual que recomende o tratamento inicial com agentes trombolíticos para a maioria dos doentes. ${ }^{15}$

- Filtro da veia cava: actualmente indicado em situações de complicações graves hemorrágicas da terapêutica médica ou no caso de contra-indicação ab- soluta para a mesma; ainda nos casos de TVP ou TEP recorrente apesar de terapêutica médica adequada. ${ }^{15}$

- Cirurgia (trombectomia): reservada para os casos graves de obstrução maciça ileo-femoral com compromisso vascular (phlegmasia cerúlea dolens), em que a trombólise está contra-indicada. ${ }^{15}$

- Meias de compressão elástica (e não «de descanso»): estudos internacionais demonstraram que o uso de meias de compressão, se diariamente, durante um longo período de tempo (mínimo de dois anos) e com compressão mínima no tornozelo de 30$40 \mathrm{mmHg}$, reduzem a incidência do síndrome pós trombótico em cerca de $50 \% .{ }^{12}$ As meias de compressão e a deambulação precoce (tolerável) devem ser recomendadas a todos os doentes na data da alta. ${ }^{14,15}$

\section{b) Tratamento a longo prazo e prevenção de recidivas:}

Após um período inicial de tratamento com heparina, há que manter o tratamento anticoagulante, na sua maioria com derivados dicumarínicos ou com heparinas de baixo peso molecular, por um período a definir em cada caso. A dose inicial recomendada para iniciar anticoagulação com dicumarínicos é de 5-10 mg/dia, sendo ajustada conforme o doseamento do INR. Nos idosos e em pacientes identificados como apresentando um elevado risco hemorrágico, a dose inicial deve ser inferior a $5 \mathrm{mg} / \mathrm{dia}^{17}$

Inicialmente, os doseamentos de INR devem ser feitos diariamente ou em dias alternados e posteriormente, semanalmente, durante algumas semanas. Quando o doente está estável, a monitorização pode ser mensal. ${ }^{2}$

Os estudos internacionais randomizados que foram efectuados e que provaram a segurança e eficácia das heparinas de baixo peso molecular no tratamento a longo prazo na TVP utilizaram dalteparina na dosagem de $200 \mathrm{UI} / \mathrm{kg} /$ dia durante um mês, seguido de 150 $\mathrm{UI} / \mathrm{kg} /$ dia ou tinzaparina $175 \mathrm{UI} / \mathrm{kg} / \mathrm{dia} .{ }^{15}$

A duração do tratamento anticoagulante tem sido alvo de múltiplos estudos prospectivos randomizados, ${ }^{1,3}$ sendo variável em função da etiologia e dos factores de risco individuais.

Resumo das actuais indicações: $:^{14,15}$

- Primeiro episódio de TVP em doente com factor de risco identificado como transitório e reversível: mí- 


\begin{tabular}{|c|c|c|c|}
\hline & $\begin{array}{l}\text { Heparina não } \\
\text { fraccionada }\end{array}$ & $\begin{array}{l}\text { Heparina de baixo } \\
\text { peso (HBPM) }\end{array}$ & $\begin{array}{l}\text { Consequências/Vantagens } \\
\text { da HBPM }\end{array}$ \\
\hline Peso molecular médio & $15000 \mathrm{Da}$ & $5000 \mathrm{Da}$ & Menos complicações \\
\hline Relação anti Xa/anti lla & Similar & Elevada & $\begin{array}{l}\text { Maior efeito antitrombótico } \\
\text { com menor risco hemorrágico }\end{array}$ \\
\hline União a proteínas plasmáticas e endotélio & Elevada & Baixa & Maior biodisponibilidade \\
\hline União a proteínas plasmáticas e plaquetas & Elevada & Baixa & Efeito mais predictível \\
\hline União a macrofagos e células endoteliais & Elevada & Baixa & Semivida mais longa \\
\hline União a produtos de activação plaquetários & Elevada & Baixa & $\begin{array}{l}\text { Menor indução de Ac antiplaquetas e } \\
\text { trombocitopénia }\end{array}$ \\
\hline Eliminação renal & Baixa & Alta & Maior vida média \\
\hline Via de administração habitual & Endovenosa & Subcutânea & Maior autonomia e menor custo \\
\hline Activação de osteoclastos & Alta & Baixa & Menor osteoporose \\
\hline Dosificação & Variável & Fixa & Não necessita de controlo laboratorial \\
\hline Monitorização & Diária & $\begin{array}{l}\text { Não necessária } \\
\text { para a maioria } \\
\text { dos doentes }\end{array}$ & \\
\hline Custo isolado do fármaco & Baixo & Elevado & \\
\hline Custos associados & Elevados & Baixos & \\
\hline
\end{tabular}

Traduzido de: Vaquero Motillo, F. Tratado de las Enfermedades Vasculares, Vol II, cap 57, Viguera Ed, 2006

nimo três meses de anticoagulação (Nota: aplicável aos doentes com trombose venosa proximal e aos doentes com TVP confinada às veias da região da perna);

- Primeiro episódio de TVP, de forma idiopática: mínimo de seis - doze meses de anticoagulação;

- Primeiro episódio de TVP com trombofilia documentada: pelo menos seis - doze meses de anticoagulação no caso de deficiência de antitrombina, proteína $\mathrm{C}$, proteína $\mathrm{S}$, factor $\mathrm{V}$ de Leiden e hiperhomocisteinémia; no caso de dois ou mais factores de trombofilia e no caso do Síndrome do Ac antifosfolipídico considerar, no mínimo, 12 meses. Em ambas as situações é de ponderar a manutenção terapêutica indefinidamente;
- Episódios recorrentes: terapêutica indefinida. Pode ser mantida com dicumarínicos ou com heparinas de baixo peso molecular, exceptuando nos doentes

\begin{tabular}{|llll|}
\hline \multicolumn{2}{l}{$\begin{array}{l}\text { QUADRO IV. Doses recomendadas para as heparinas de baixo peso } \\
\text { molecular mais utilizadas no tratamento inicial da TVP }\end{array}$} \\
\begin{tabular}{llll} 
Princípio Activo & Nome Comercial & $\begin{array}{l}\text { Dosificação para } \\
\text { administração } \\
\text { cada } 12 \mathrm{~h}\end{array}$ & $\begin{array}{l}\text { Dosificação para } \\
\text { administração } \\
\text { cada 24h }\end{array}$ \\
\hline Dalteparina & Fragmin & $100 \mathrm{UI} / \mathrm{kg}$ & $200 \mathrm{UI} / \mathrm{kg}$ \\
\hline Enoxiparina & Lovenox & $100 \mathrm{UI} / \mathrm{kg}$ & $150 \mathrm{UI} / \mathrm{kg}$ \\
\hline Nadroparina & Fraxiparina & $85 \mathrm{UI} / \mathrm{kg}$ & $170 \mathrm{UI} / \mathrm{kg}$ \\
\hline Tinzaparina & Innohep & - & $175 \mathrm{UI} / \mathrm{kg}$ \\
\hline
\end{tabular}
\end{tabular}

Legenda: UI: unidades internacionais anti-Xa

Traduzido de:Vaquero Motillo, F. Tratado de las Enfermedades Vasculares, Vol II, cap 57, Viguera Ed, 2006 
com cancro, em que a eficácia das últimas é superior à terapêutica oral

As contra-indicações para a anticoagulação são:

- AVC hemorrágico nos últimos três meses

- Cirurgia major recente

- Traumatismo significativo recente

- Hemorragia activa (contra-indicação absoluta)

- Diátese hemorrágica identificada

- Neoplasia intracraneana

- Gravidez

Nestes doentes devem ser ponderadas outras opções terapêuticas.

Em relação aos doentes que tomam dicumarínicos, há que recordar que estes fármacos têm múltiplas interacções farmacológicas, (inclusivamente com a dieta alimentar, sendo de evitar o elevado consumo de produtos hortícolas verdes) e que suscitam dúvidas sobre qual a melhor alternativa, no caso de terem de se efectuar procedimentos tão simples como um tratamento dentário. No entanto, na maioria dos doentes, não é necessária alteração da intensidade de terapia anticoagulante para proceder a tratamentos dentários, mas podem existir diferenças entre a incidência de hemorragias após os procedimentos. Se existir necessidade de limitar o sangramento local, recomenda-se a utilização local de elixir de ácido tranexâmico ou ácido épsilon-aminocapróico. ${ }^{17}$

Para os doentes que necessitam de efectuar cirurgia existem tabelas de estratificação de risco do doente em baixo, médio e elevado risco trombótico e recomendações clínicas adequadas a cada caso.

Seguimento no ambulatório:

Segundo a bibliografia consultada, ${ }^{3}$ a maioria dos doentes com TVP proximal dos membros inferiores confirmada pode ser tratada com segurança em ambulatório. Os critérios de exclusão são os seguintes:

- Suspeita de tromboembolismo pulmonar concomitante;

- Co-morbilidade cardiovascular ou pulmonar significativa;

- TVP ileofemoral;

- Doentes em que está contra-indicada a anticoagulação;

- Alterações genéticas da coagulação;

- Gravidez;

- Obesidade mórbida (>150 Kg);
- Insuficiência renal com creatinina superior a $2 \mathrm{mg} / \mathrm{dl}$;

- Impossibilidade de prestar cuidados no ambulatório;

- Impossibilidade de seguimento das indicações clínicas;

- Sem abrigo ou residentes em áreas geográficas afastadas dos cuidados de saúde;

- Sem contacto telefónico disponível;

- Doentes ou familiar responsável que recusam expressamente o tratamento ambulatório.

\section{CONCLUSÕES}

A TVP é uma doença médica de origem multifactorial, que consiste na oclusão de um vaso da circulação venosa profunda por um trombo. Para que este fenómeno ocorra, é necessário estarem presentes os três elementos da Tríade de Virchow: estase, hipercoagulabilidade e lesão endotelial que, interagindo entre si, desencadeiam as múltiplas alterações a nível do vaso sanguíneo.

O diagnóstico de TVP não é fácil nem preciso, devendo basear-se em tabelas de estratificação de risco e necessita de ser confirmado por meios auxiliares de diagnóstico, nomeadamente doseamentos analíticos e/ou exames imagiológicos. Nos meios complementares imagiológicos, o método de eleição é a venografia, que tem vindo a ser substituída pelo exame ecográfico vascular com doppler, devido à sua sensibilidade e especificidade elevadas, embora dependente da experiência do utilizador. Infelizmente, no contexto diário da prática clínica do Médico de Família, verificamos que a maioria dos doentes com suspeita de TVP necessitam de ser referenciados a uma urgência hospitalar, para realização de análises e/ou doppler. A limitação económica decorrente da não comparticipação destes estudos imagiológicos acaba por não deixar grande alternativa ao Médico de Família.

$\mathrm{O}$ tratamento precoce permite prevenir a extensão do trombo, o tromboembolismo venoso e a síndrome pós-trombótica, melhorando o prognóstico do doente.

O tratamento deve incluir anticoagulação, inicialmente recorrendo a heparina não fraccionada ou de baixo peso molecular e, posteriormente, a dicumarínicos, com duração variável em função do caso.

No caso de contra-indicações para anticoagulação, há várias opções médicas e cirúrgicas disponíveis. 
O factor de risco mais importante para TVP é um episódio prévio da mesma, pelo que os doentes necessitam de um acompanhamento adequado, envolvendo, também, a sua educação, nomeadamente na evicção de imobilização prolongada, esclarecimento dos riscos associados ao uso de estrogénios no caso das pacientes do sexo feminino, e o uso continuado de meias de compressão elástica.

\section{REFERÊNCIAS BIBLIOGRÁFICAS}

1. Vaquero Morillo F. Capítulo 57: Diagnóstico. In: Tratado de las Enfermedades Vasculares., Vol II, cap 57, Barcelona: Viguera Editores; 2006. p. 1031-40.

2. Schreiber D. Deep Venous Thrombosis and thromboflebitis. Disponível em: http://emedicine.medscape.com [acedido em 12/07/2010].

3. Patel K, Feied C. Deep Venous Thrombosis. Disponível em: http://emedicine.medscape.com [acedido em 13/07/2010].

4. Anderson FA Jr, Spencer FA. Risks factors for venous thromboembolism. Circulation 2003 Jun 17; 107 (23 Suppl 1): 9-16.

5. Rocha E, Lecumberri R, Feliu J. Trombosis y embarazo. In: Salinas e, editor. Abordaje práctico de las alteraciones hematológicas en la mujer embarazada y en el neonato. Madrid: Acción Médica; 2004. p. 133-56.

6. Eklof B. Air travel related venous thromboembolism: an existing problem that can be prevented. Cardiovasc Surg 2002 Apr; 10 (2): 95-7.

7. MansilhaA. Polimorfismos Genéticos e doença tromboembólica venosa. Rev Angiologia Cir Vasc 2005; 1: 15-23.

8. Tripodi A. A review of the clinical and diagnostic utility of laboratory tests for the detection of congenital thrombofilia. Semin Thromb Hemost 2005 Feb; 31 (1): 5-10.

9. Misita CP, Moll S. Antiphospholipid antibodies, Circulation 2005 Jul 19; 112 (3): e39-44.

10. Anand SS, Wells PS, Hunt D, Brill-Edwards P, Cook D, Ginsberg JS. Does this patient have deep vein thrombois? JAMA 1998 Apr 8; 279 (14): 1094-9.
11. Labropoulos N, Tassiopoulos AK. Vascular diagnosis ofn venous thrombosis. In: Mansour MA, Labropoulos N, editors. Vascular Diagnosis. Philadelphia, PA: Elsevier Saunders; 2005.

12. Páramo Fernández JA. Diagnostico de hipercoagulabilidad. Rev Clin Esp 2001; 201:30-2.

13. Ginsberg JS, Hirsh J, Julian J, Vander LaandrVries M, Magier D, MacKinnon $B$, et al. Prevention and treatment of postphlebitic syndrome: results of a 3-part study. Arch Inter Med 2001 Sep 24; 161 (17): 2105-9.

14. Vaquero Morillo F. Capítulo 56: Generalidades e historia natural. In: Tratado de las Enfermedades Vasculares. Barcelona: Viguera Ediciones; 2006. p. 1013-30.

15. Buller HR, Agnelli G, Hull RD, Hyers TM, Prins MH, Raskob GE. Antithrombotic therapy for venous thromboembolic disease: the Seventh ACCP Conference on Antithrombotic and Thrombolytic Therapy. Chest 2004 Sep; 126 (3 Suppl): 401S-428S.

16. Warkentin TE, Greinacher A. Heparin-induced Thrombocytopenia: recognition, treatment, and prevention: the Seventh ACCP Conference on Antithrombotic and Thrombolytic Therapy. Chest 2004 Sep; 126 (3 Suppl): 311S-337S.

17. Ansell J, Hirsh J, Poller L, Bussey H, Jacobson A, Hylek E. The pharmacology and management of the vitamin K antagonists: the Seventh ACCP Conference on Antithrombotic and Thrombolytic Therapy. Chest 2004 Sep; 126 (3 Suppl): 204S-233S.

\section{CONFLITOS DE INTERESSE}

A Autora declara não existirem conflitos de interesse na execução e redacção do artigo.

\section{ENDEREÇO PARA CORRESPONDÊNCIA}

Susana Dias Antunes

Unidade de Saúde Familiar Condes da Lousã

Praceta Condes da Lousã, Damaia, 2720 - Amadora

E-mail: santunes@sapo.pt

Recebido em 27/07/2010

Aceite para publicação em 12/10/2010

\section{ABSTRACT}

\section{DEEP VENOUS THROMBOSIS: MYTHS AND REALITY}

Deep Venous Thrombosis (DVT) is a medical condition with an annual incidence of 117 cases for 100.000 in the USA, and is one of the main causes of death in hospitalized patients.

Its etiology is multifactorial, and multiple factors must interact for a thrombus to form inside a blood vessel.

Its diagnosis, risk stratification and the referral to a vascular specialist for diagnostic confirmation and adequate treatment allow for a decrease in mortality and morbility.

This article intends to be a brief but precise revision of the aetiology, diagnostic criteria and treatment of DVT.

Key-words: Deep Vein Thrombosis; Venous Disease; Heparin, Low Molecular - Weight; Anticoagulation; Post-thrombotic Syndrome. 